\title{
Scientific Uncertainty and Climate Change: Part II. Uncertainty and Mitigation
}

\author{
Stephan Lewandowsky • James S. Risbey • \\ Michael Smithson • Ben R. Newell
}

November, 2013

\begin{abstract}
In public debate surrounding climate change, scientific uncertainty is often cited in connection with arguments against mitigative action. This article examines the role of uncertainty about future climate change in determining the likely success or failure of mitigative action. We show by Monte Carlo simulation that greater uncertainty translates into a greater likelihood that mitigation efforts will fail to limit global warming to a target (e.g., $2^{\circ} \mathrm{C}$ ). The effect of uncertainty can be reduced by limiting greenhouse gas emissions. Taken together with the fact that greater uncertainty also increases the potential damages arising from unabated emissions (Lewandowsky, Risbey, Smithson, Newell, \& Hunter, in press), any appeal to uncertainty implies a stronger, rather than weaker, need to cut greenhouse gas emissions than in the absence of uncertainty.
\end{abstract}

Keywords Uncertainty · mitigation failure · decision making

\section{Introduction}

Uncertainty is an integral part of science. Although the scientific community has sought to deal with uncertainty in various ways (e.g., Intergovernmental Panel on Climate Change, 2005; Narita, 2012), it is often highlighted in public debate to argue for the delay of politically-contentious actions that may entail a regulatory burden on industry (Kim, 2011; Nisbet, 2009). For example, public debate about the adverse health consequences of tobacco continued for decades after the medical community had considered the scientific case largely settled (e.g., Brownell \& Warner, 2009; Michaels \& Monforton, 2005; Nilsson, Beaglehole, \& Sauerborn, 2009; Proctor, 2004, 2008; P. Smith et al., 2011). Appeals to uncertainty constituted a key ingredient of arguments put forward against a public-health response (e.g., Freudenburg, Gramling, \& Davidson, 2008; Oreskes \& Conway, 2010; P. Smith et al., 2011).

University of Western Australia and University of Bristol E-mail: stephan.lewandowsky@bristol.ac.uk · CSIRO Marine and Atmospheric Research, Hobart, Tasmania · Australian National University · University of New South Wales 
The effectiveness of appeals to uncertainty derives in part from the fact that risk judgments are known to be influenced by psychological, cultural, and political factors (e.g., Kahan, Slovic, Braman, \& Gastil, 2006; Slovic, 1999). To illustrate, Kahan, Braman, Slovic, Gastil, and Cohen (2009) provided participants with information about the risks and benefits of nanotechnology and then solicited judgments of the relative risk and benefits of the new technology. Responses were found to diverge according to people's worldview: People who lean towards a hierarchical and market-oriented view of society tended to downplay risks and focused on the benefits, whereas people with a more egalitarian worldview emphasized the risks over the benefits. Thus, instead of uniformly "educating" people, the provision of information about a new technology led to enhanced polarization of people's views.

Those cultural and psychological factors also enter into the conventional formal tools with which risky policy decisions are informed, such as cost-benefit-analysis (CBA; e.g., Garnaut, 2011; Nordhaus, 2010; Stern, 2007) or the precautionary principle (e.g., Vlek, 2010a, 2010b). For example, the choice of economic discount rate (i.e., the rate at which future costs are reduced to render them comparable to today's expenditure) can alter anticipated costs by many orders of magnitude (Sterner \& Persson, 2008). Because the discount rate can vary across a range of plausible values, CBA provides no escape from the inherently subjective nature of risk judgments and decision making under uncertainty (for more details, see Lewandowsky et al., in press).

This article explores the way in which uncertainty about future changes in climate relate to the likely consequences of mitigative action. To foreshadow briefly, here we show that greater uncertainty about climate sensitivity increases the likelihood that a given carbon budget will be exceeded, which we refer to as "mitigation failure." We also show that the adverse implications of uncertainty can be reduced by curtailing greenhouse gas emissions.

\section{Consequences of Uncertainty: Risks and Outcomes}

We operationalize uncertainty as the standard deviation or variance of the estimate of future global warming in response to a doubling of greenhouse gas emissions (following precedent; e.g., Padilla, Vallis, \& Rowley, 2011). Given that our focus here is on mitigation, and thus on moderately short time spans of around a century or less, we are concerned with peak sensitivity (Raupach et al., 2011). Peak climate sensitivity (PCS) is defined as the peak warming that is observed in response to the radiative forcing at that time; for details see Raupach et al. (2011). ${ }^{1}$ To prevent ambiguity, we refer to the uncertainty about our knowledge of peak climate sensitivity as uncertaintyPCS from here on, with other subscripts introduced where necessary to identify other manifestations or sources of uncertainty.

1 PCS, like all other climate sensitivity measures such as the transient climate response (TCR; the expected increase in global temperatures at the time of doubling of $\mathrm{CO}_{2}$ from pre-industrial levels after a $1 \% /$ year increase), is a ratio between expected warming and forcing. Those ratios have been shown to be constant in a linear climate system if forcings grow exponentially (Raupach, 2013), implying that an analysis based on PCS generalizes to other measures such as TCR. 
Our approach relies on relating the magnitude of uncertaintypCS at a purely ordinal level to the associated outcomes. Thus, we are concerned with answering ordinal questions such as what greater (or lesser) uncertaintypCs would imply, rather than seeking point estimates of risks or outcomes (e.g., Webster et al., 2003). Answering such ordinal questions can address uncertainty-based argumentations (Freudenburg et al., 2008; Freudenburg \& Muselli, 2013): For example, if greater uncertainty ${ }_{\mathrm{PCS}}$ were associated with greater likelihood of harm from a potential path of action, then one cannot legitimately argue against that path of action by claiming that there is "too much" scientific uncertainty.

Using that ordinal approach, we showed in a companion article Lewandowsky et al. (in press) that greater uncertainty about the climate translates into greater expected damages from unabated greenhouse emissions. Because the companion paper was concerned with the long-term consequences of unabated emissions it considered equilibrium climate sensitivity (ECS) instead of peak sensitivity (PCS). The result that greater uncertainty yields greater damages arose from the fact that the cost function that relates global temperature increases to economic damages is pervasively assumed to be convex (i.e., accelerating; see, e.g., Tomassini et al., 2010). For mathematical reasons, a convex cost function necessarily implies that greater uncertainty about global temperature increases (captured by uncertainty ${ }_{\mathrm{ECS}}$ ) translates into greater expected damage costs. The same result was also obtained without making any assumptions about an economic cost function, by considering the impact of uncertainty surrounding future sea level rise. As uncertainty about future sea level rises increases, so does the need for additional protective measures (e.g., increasing the height of dams and dikes) to keep the risk of inundation constant. (For details, see Hunter, 2012, and Lewandowsky et al., in press).

Whereas the companion article Lewandowsky et al. (in press) focused on the consequences of unabated emissions, we now apply the same ordinal approach to examining the impact of uncertainty on the likely success of mitigation efforts. We adopt a "safe-minimum-standard" approach by examining the risk of exceeding an agreed threshold increase in global temperatures. We do not argue in a favor of one specific threshold over another but simply assume the existence of an agreed value.

\section{Uncertainty and Mitigation of Climate Change}

\subsection{The Limited Carbon Budget}

In order to have a reasonable chance ( $66 \%$ or more) of limiting future temperature increases to $2^{\circ} \mathrm{C}$ - an upper bound on warming that is considered "safe" by many governments - atmospheric $\mathrm{CO}_{2}$ levels must stabilize somewhere between $415 \mathrm{ppm}$ and 460 ppm (Rogelj et al., 2011). Because $\mathrm{CO}_{2}$ remains in the atmosphere for centuries (Solomon, Plattner, Knutti, \& Friedlingstein, 2009), this upper limit of atmospheric concentrations also places an upper bound on the total amount of carbon that humanity can emit: This amount represents the total "budget" of carbon that is available to "spend," from the beginning of industrialization until, effectively, an infinitely distant time from now. This total available budget is estimated to be in the vicinity of $1,000 \mathrm{Gt}$ carbon $(\mathrm{C})$, equivalent to $3,664 \mathrm{Gt}$ 
$\mathrm{CO}_{2}$ (Raupach et al., 2011), of which roughly 50\% (531 Gt C) had already been expended by 2008 on fossil fuels and land use combined (Raupach et al., 2011). ${ }^{2}$

The implications of this fixed budget, which limits the sum total of all remaining future emissions to approximately $500 \mathrm{Gt} \mathrm{C}$, are shown in Figure 1. The figure shows various hypothetical emissions trajectories - i.e., annual global greenhouse gas emissions over time- based on assumptions about annual emissions growth and the rate of emission cuts after mitigation commences.

Consider first the left panel (A), which shows four emissions trajectories, all of which keep total emissions (including the historical liabilities arising from past emissions) to 1,000 Gt C. The principal difference between trajectories is the time at which mitigation efforts commence, which varies from 2011 to 2026 . Until the commencement of mitigation, emissions are assumed to grow at a realistic annual rate of $3 \%$ (and at a gradually reducing rate for some time after commencement of mitigation owing to technological inertia; see Equations 9 and 10 in Raupach et al., 2011).

The panel emphasizes that the longer carbon emissions are permitted to grow unabated, the steeper are the cuts that are required to keep warming from exceeding an agreed threshold. If mitigation had commenced in 2011, the required annual rate of emissions reduction would be $6 \%$ per year, whereas a delayed commencement in 2021 or 2026 would mandate ongoing annual cuts of $11 \%$ and $17 \%$, respectively. This budgetary principle holds irrespective of the magnitude of the threshold; a higher threshold merely postpones the problem because the budget increases, but the relationship between delay and rigor of required emission cuts is unaffected.

Experience with large structural changes suggests that the cost of mitigation increases with the required annual mitigation rate: Whereas a few percentage points might be achievable, at least initially, by efficiency measures and minor adjustments of consumer behavior, steeper cuts require the introduction of new technologies and radical structural changes at greater cost. In confirmation, a retrospective analysis of the last 7 years of unabated emissions reveals that future mitigation efforts have become more difficult and more expensive: In 2004, Pacala and Socolow argued that with existing technologies, humanity already possessed "... the fundamental scientific, technical, and industrial know-how to solve the carbon and climate problem for the next half-century" (2004, p. 968). At the time, Pacala and Socolow provided a portfolio of 7 so-called "wedges" that, when used as an ensemble, could avoid doubling of atmospheric $\mathrm{CO}_{2}$ compared to pre-industrial levels. Those "wedges" included more efficient buildings, reduced reliance on cars, greater efficiency of power plants, and so on. Revisiting this issue 7 years later, Socolow (2011) concluded that now 9, rather than 7 , wedges were required to stabilize $\mathrm{CO}_{2}$ emissions, and that the delay of mitigation would produce nearly $0.5^{\circ} \mathrm{C}$ of additional warming. ${ }^{3}$

2 For tractability and ease of exposition, all estimates relating to the carbon budget in this article are taken from Raupach et al. (2011). Those estimates are consonant with other reviews (e.g., Rogelj et al., 2011). Short-lived gasses such as hydrofluorocarbons do not contribute to the total budget (S. M. Smith et al., 2012).

3 Pacala and Socolow (2004) and Socolow (2011) considered stabilization of emissions only (i.e., preventing further growth), rather than the cuts to emissions that are necessary to stay within a finite carbon budget. Their analysis thus underestimates the consequences of delayed mitigation, amplifying the point that delay enhances costs. 


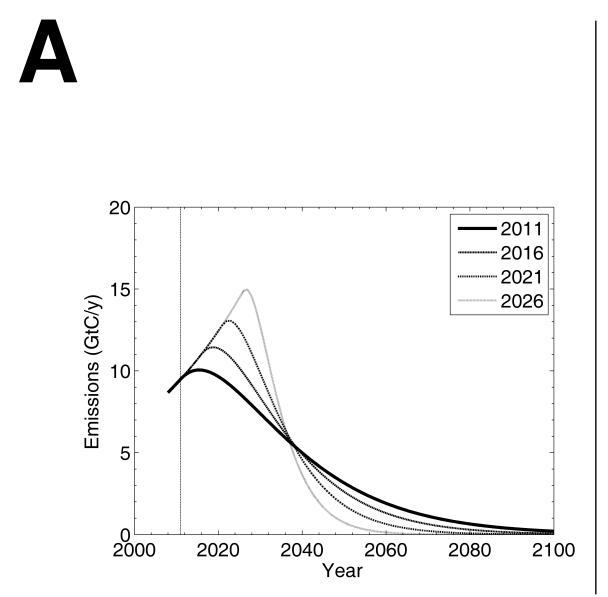

\section{B}

Fig. 1 Required emissions trajectories that limit total cumulative carbon emissions from fossil fuel and land use combined (including historical emissions) to 1,000 Gt C. All trajectories are smooth and computed using Equations 9 and 10 in Raupach et al. (2011). Trajectories commence in 2008 and differ with respect to the starting point of mitigation as shown in the legend. Panel A shows trajectories for 4 different times at which mitigation commences, all of which achieve a total budget of $1,000 \mathrm{Gt}$ C. Panel B shows another emissions trajectory that achieves the same total budget but mitigation is delayed to a time (2033) at which emissions must cease virtually instantaneously. There exists no smooth emissions trajectory with a later commencement of mitigation (in whole years) that complies with the same total budget. Vertical lines indicate the present, defined here as 2011.

Nonetheless, the pattern shown in Figure 1A need not, by itself, present an indisputable impetus to commence mitigation sooner rather than later: With a conveniently chosen discount rate, the extra 15 years that separate a $17 \%$ annual mitigation rate from a $6 \%$ rate (i.e., 2026 vs. 2011) may make the additional cost associated with delayed mitigation more attractive (e.g., a discount rate of around $4.5 \%$ will accommodate a doubling of mitigation costs after 15 years).

It is therefore important to consider panel B in Figure 1, which takes the implications of delaying mitigation to an extreme. This panel contains an emissions trajectory which maximally delays the commencement of mitigation (to 2033) while still complying with the total budget of 1,000 Gt C. No further delay (by whole years) is possible without exceeding that budget. The notable feature of the trajectory in Figure 1B is that emissions must cease virtually instantaneously when mitigation commences. Virtually instant cessation of emissions is technologically and logistically so unlikely as to be considered impossible - and this impossibility cannot be overcome by the choice of discount rate. We therefore characterize the trajectory in Figure $1 \mathrm{~B}$ as representing a failure of mitigation, and our analysis of uncertainty focuses on the likelihood of such a failure, thereby sidestepping potential counter-arguments based on the discount rate.

\subsection{Uncertainty and the Carbon Budget}

Our analysis of the role of uncertainty ${ }_{\mathrm{PCS}}$ in mitigation begins by considering Figure 2 which relates the expected extent of global warming to the total cumulative 


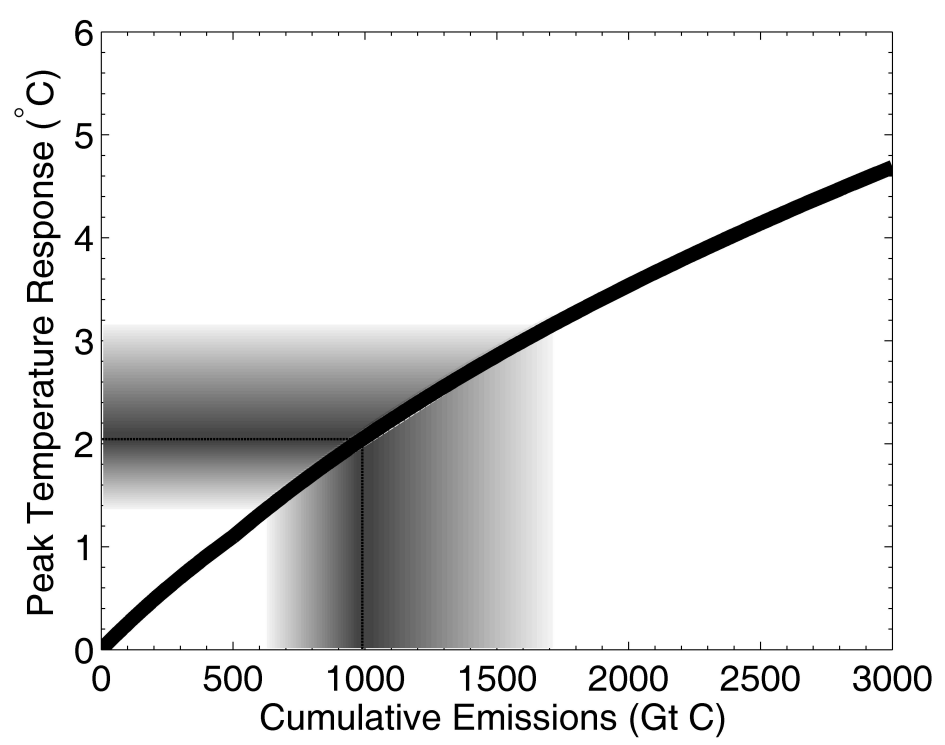

Fig. 2 Expected peak temperature response of the climate system relative to pre-industrial times (in ${ }^{\circ} \mathrm{C}$ ) as a function of all-time total cumulative emission (in Gt $\mathrm{C}$ ). The figure was created using Equations 8 and 13 in Raupach et al. (2011). The temperature response assumes a (peak) climate sensitivity of $2.75^{\circ} \mathrm{C}$ (i.e., $\lambda_{p}=.74 \mathrm{~K} \mathrm{~W}^{-1} \mathrm{~m}^{2}$ ); confidence bounds on the temperature response are provided in Figure 5. The horizontal line and the surrounding uncertainty band (arbitrarily chosen but representing the expected asymmetric upper tail in the temperature response) illustrate the relationship between uncertaintyPCS - and hence uncertainty ${ }_{\mathrm{TR}}$ about the temperature response- and the corresponding uncertainty ${ }_{\mathrm{B}}$ about the associated carbon budget (vertical line and associated uncertainty band). See text for details.

emissions of greenhouse gases (primarily $\mathrm{CO}_{2}$ but also including other forcings). Note that unlike Figure 1, which shows annual emissions, Figure 2 shows cumulative emissions; that is, the sum total emitted from the onset of industrialization until an infinitely distant point in time. The figure links up with the preceding discussion by noting that cumulative emissions must not exceed 1,000 Gt $\mathrm{C}$ for warming to remain below $2^{\circ} \mathrm{C}$.

Uncertainty ${ }_{\mathrm{PCS}}$ enters into this relationship between cumulative emissions and temperature response via the fact that the expected global temperature increase is a multiple of climate sensitivity. Climate sensitivity is defined as the temperature increase in response to a doubling of $\mathrm{CO}_{2}$ compared to preindustrial levels; the value of the temperature response function in Figure 2 is therefore equal to the climate sensitivity for a budget that corresponds to a doubling of preindustrial atmospheric $\mathrm{CO}_{2}$ levels, and it is a multiple (or fraction) of climate sensitivity elsewhere.

It follows that uncertainty ${ }_{\mathrm{PCS}}$ maps directly, via a proportional constant, into uncertainty about the temperature response, called uncertaintyтR from here on. Uncertainty $_{\text {TR }}$ can be translated into uncertainty ${ }_{B}$ about the carbon budget via the response function shown in Figure 2. Specifically, any uncertaintyтr about a point on the ordinate (temperature response) can be "reflected" into a cor- 
responding uncertainty ${ }_{B}$ along the abscissa (cumulative emissions); this is illustrated in the figure by the horizontal line (drawn at $2^{\circ} \mathrm{C}$ for convenience) and its surrounding arbitrary uncertainty ${ }_{\mathrm{TR}}$ band. That temperature response, and its associated uncertainty ${ }_{\mathrm{TR}}$, is reflected into the vertical line with its own associated uncertainty $_{B}$ band along the cumulative emissions axis.

To investigate this relationship between uncertainties in the temperature response and in total emissions we conducted a Monte Carlo simulation. Values of climate sensitivity were sampled from a lognormal distribution with mean $\mu_{L}=2.75^{\circ} \mathrm{C}$ and standard deviations $\left(\sigma_{L}\right)$ ranging from $.26^{\circ} \mathrm{C}$ to $1.66^{\circ} \mathrm{C}$ in 7 steps. The distribution of climate sensitivity is known to have a "fat" upper tail (for discussion, see, e.g., Roe \& Baker, 2007). To capture that fact we sampled sensitivities from a lognormal distribution, without however being committed to that precise shape of the distribution (cf. Lewandowsky et al., in press). ${ }^{4}$

For each level of uncertaintypCS (i.e., standard deviation $\sigma_{L}$ of the lognormal distribution), 100,000 realizations of sensitivity were sampled, and for each realization a corresponding carbon budget was obtained - using the equations underlying Figure 2 - that limited warming to $2^{\circ} \mathrm{C}$. Figure 3 shows two snapshots of this process (using only 1,000 realizations for illustration), for two levels of uncertainty $\mathrm{PCS}$ $\left(\sigma_{L}=0.37\right.$ and $\sigma_{L}=1.17$ in panels $\mathrm{A}$ and $\mathrm{B}$, respectively). In each panel, the histogram at the top shows the simulated distribution of climate sensitivities: Note that the mean $\mu_{L}$ is identical between panels $\mathrm{A}$ and $\mathrm{B}$ and only the standard deviation $\sigma_{L}$ of the distribution changes. (See Lewandowsky et al., in press, for a justification of using a mean-invariant analysis of uncertainty.) The histograms in the middle show the associated distributions of carbon budgets, and the functions at the bottom relate each realization of sensitivity to a corresponding budget.

Two observations can be made with respect to Figure 3: First, as climate uncertainty ${ }_{P C S}$ increases, the variance of the distribution of maximum acceptable carbon budgets (uncertaintyB) also increases. Second, increasing uncertainty PCS $_{\mathrm{B}}$ changes the shape of the carbon-budget distribution, with an increasing number of small carbon budgets accompanied by a smaller number of very large budgets.

Figure 4 expands on those snapshots by summarizing the simulated distributions of carbon budgets across all levels of uncertaintypCS. Specifically, each set of points for a given uncertainty ${ }_{P C S}$ across both panels in Figure 4 summarizes a carbon-budget distribution; thus, the center histograms in panels A and B of Figure 3 correspond, respectively, to the data points at $\sigma_{L}=0.37$ and $\sigma_{L}=1.17$ across both panels of Figure 4.

When uncertainty ${ }_{\mathrm{PCS}}$ is minimal (left-most data points in Figure 4), the expected budget is around 1,000 Gt $\mathrm{C}$ on average, which is of course the value associated with $2^{\circ} \mathrm{C}$ peak warming in Figure 2 - as it must be because the simulation was designed to examine budgets that keep warming below the $2^{\circ} \mathrm{C}$ threshold. Note also that the distribution of carbon budgets remains sharply peaked when uncertainty ${ }_{\mathrm{PCS}}$ is minimal, as indicated by the fact that the mean, median, and first quartile (25th percentile; Q25) are clustered closely together.

4 Lognormal distributions are often characterized by the parameters of the underlying Gaussian distribution, $\mu_{G}$ and $\sigma_{G}$. They are related to the equivalent parameters of the lognormal distribution via $\mu_{L}=\exp \left(\mu_{G}+.5 \sigma_{G}^{2}\right)$ and $\sigma_{L}=\exp \left(\mu_{G}+.5 \sigma_{G}^{2}\right) \times \sqrt{\exp \left(\sigma_{G}^{2}\right)-1}$. All parameter values characterizing lognormal distributions in this article are provided in terms of $\mu_{L}$ and $\sigma_{L}$. 

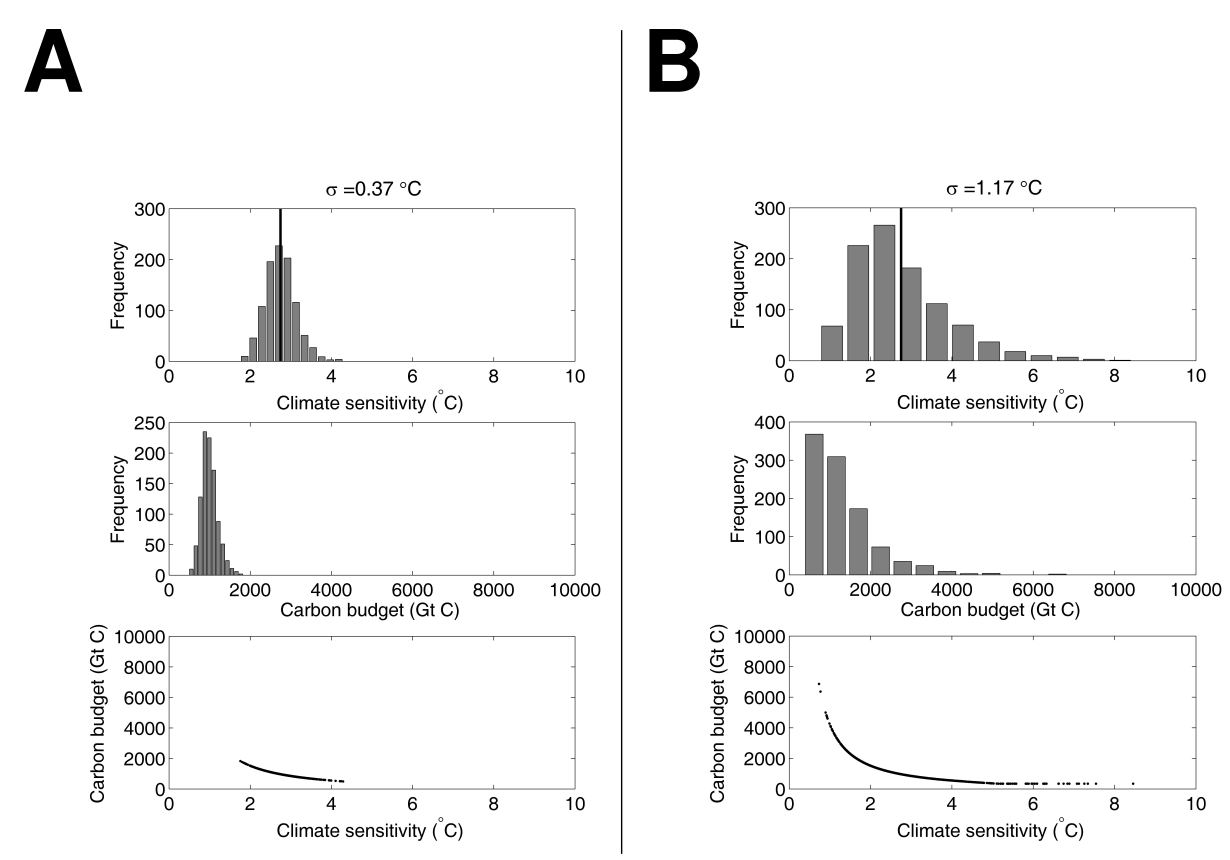

Fig. 3 Two snapshots of a Monte Carlo simulation relating uncertainty ${ }_{\mathrm{PCS}}$ in climate sensitivity $\left(\sigma_{L}=.37\right.$ in panel $\mathrm{A}$ and $\sigma_{L}=1.17$ in panel $\left.\mathrm{B}\right)$ to the carbon budget required to limit warming to $2^{\circ} \mathrm{C}$. Histograms at the top show the simulated distribution of climate sensitivity (whose mean is indicated by the vertical line), and histograms in the center show the associated carbon budgets. Carbon budgets cannot fall short of the historical emissions of $346 \mathrm{Gt} \mathrm{C}$ (i.e., carbon emissions from fossil fuels up to 2008; land-use related emissions are omitted for simplicity.) Functions at the bottom relate sampled sensitivities to budgets.

When uncertaintyPCS increases, the mean total carbon budget also increases (top panel of Figure 4). That is, the greater our uncertainty about climate sensitivity, the greater the expected (average) carbon budget that is available for expenditure. The reason for this arises from the form of the function that relates cumulative emissions to temperature (Figure 2): It is concave (i.e., increasing but with decelerating slope) and therefore, by a theorem known as Jensen's inequality (e.g., Jensen, 1906), on average greater uncertainty about the climate yields a larger carbon budget. ${ }^{5}$

However, the mean of the simulated carbon budgets does not provide a complete picture, because the shape of the budget distribution is of particular relevance. The remaining lines in the top panel of Figure 4 describe the shape of those budget distributions across levels of uncertainty ${ }_{\mathrm{PCS}}$ by plotting their median, the first quartile (Q25), and finally the minimum. The fact that the mean, median, and Q25 diverge as uncertainty ${ }_{\mathrm{PCS}}$ increases reflects the fact that the uncertainty

5 Note that this involves an inference from the ordinate to the abscissa in Figure 2; if the function were inverted to show the budget as a function of a temperature response, which is the more routine formulation of Jensen's inequality, then the function would be convex. For a detailed discussion of the implications of increasing uncertainty in the presence of convex functions, see Lewandowsky et al. (in press). 

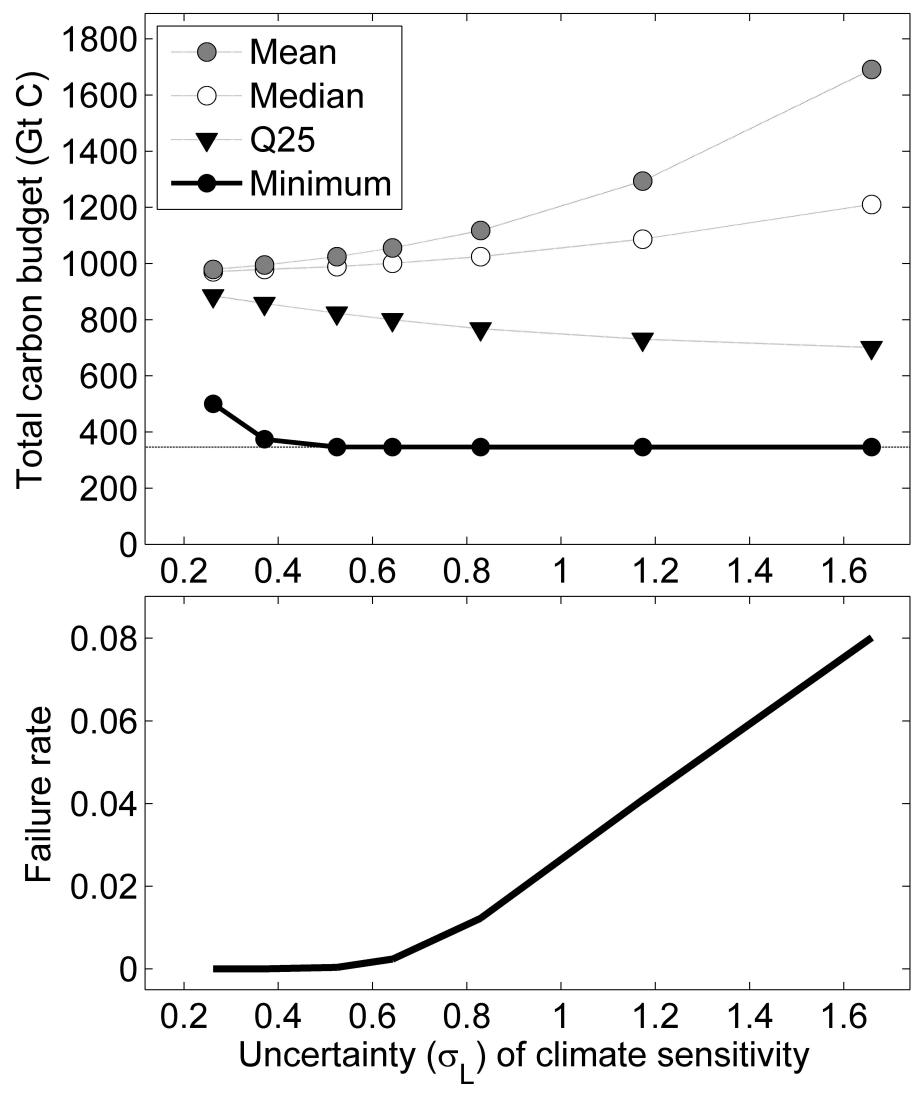

Fig. 4 Effects of increasing climate-sensitivity uncertainty ${ }_{\mathrm{PCS}}$ (standard deviation, $\sigma_{L}$, in ${ }^{\circ} \mathrm{C}$ ) on the distribution of carbon budgets to limit warming to $2^{\circ} \mathrm{C}$ (top panel) and associated failure rate of mitigation (bottom panel). The failure rate is defined as the proportion of carbon budgets that fall below the total emissions to date of $346 \mathrm{Gt} \mathrm{C}$. The summary statistics in the top panel characterize the distribution of carbon budgets across the 100,000 Monte Carlo realizations for each level of climate uncertainty. Q25 refers to the 25th percentile or first quartile, and the dotted horizontal line indicates historical emissions. Note that while increased uncertainty increases the mean budget, the median increases only slightly and the first quartile decreases. It follows that there is a greater than $25 \%$ and near $50 \%$ probability that greater uncertainty will translate into a carbon budget lower than the mean expectation.

of the resulting acceptable carbon budgets also increases; compare the two center histograms in Figure 3 for an illustration.

Figure 4 also shows that the minimum budget-associated with the greatest sampled sensitivities; see bottom panels in Figure 3-decreases as uncertaintyPCS increases, before it flattens out as it hits the thin horizontal line. The reason that the minimum budget rapidly reaches an asymptote is because no carbon budget can be lower than the total carbon already emitted (i.e., $346 \mathrm{Gt} \mathrm{C}$ from fossil fuels). Those historical emissions cannot be undone and have a notable implication: Whenever a carbon budget falls at or below that lowest possible value, mitigation has already failed. That is, even if emissions were to cease completely and virtually 
instantaneously - as in Figure 1B - the target maximum warming has already been exceeded.

The implications of this problem are illustrated further in the bottom panel of Figure 4, which shows the proportion of simulated carbon budgets (out of 100,000 for each level of uncertainty ${ }_{\mathrm{PCS}}$ ) that fall at or below the minimum dictated by historical emissions. The panel thus records the rate of mitigation failures; that is, the probability that the immediate urgency of required emission cuts exceeds technological, logistical, and most certainly also political, constraints. It can be seen that as uncertainty ${ }_{\mathrm{PCS}}$ increases, so does the failure rate: The more uncertainty there is about the climate, the more likely it is that we have already passed the point beyond which it is no longer possible to limit warming to $2^{\circ} \mathrm{C}$.

Note that the maximum of our simulated uncertaintiesPCS, $\sigma_{L}=1.66$, meshes well with empirical estimates of the standard deviation of the distribution of climate sensitivities (e.g., $\sigma=1.7$; Annan \& Hargreaves, 2006). The fact that a failure rate of $8 \%$ can arise with this plausible value of climate uncertainty ${ }_{\mathrm{PCS}}$ should therefore give rise to concern. This $8 \%$ failure rate can be put into context by comparing it to another "dread" risk, such as nuclear reactor accidents (Slovic, 1987): It is unlikely that the public - or indeed governments - would tolerate nuclear power if it came with an $8 \%$ annual risk of a meltdown somewhere in the world. (The three major nuclear accidents to date, Three Mile Island, Chernobyl, and Fukushima, occurred over a nearly 60-year history of nuclear power generation, pointing to an annual failure rate of below $8 \%$. Those three accidents were nonetheless sufficient for nuclear power to be abandoned in at least some countries; Jahn \& Korolczuk, 2012.)

\subsection{Summary: Uncertainty and Mitigation of Climate Change}

Our analysis examined the effects of climate uncertainty ${ }_{\mathrm{PCS}}$ on the likely size of the carbon budget and yielded three main findings: First, greater uncertainty ${ }_{\mathrm{PCS}}$ yields a greater carbon budget on average compared to what would be expected if climate sensitivity were known with great precision.

Second, there is a substantial likelihood-i.e., in excess of $25 \%$ and approaching 50\%; see median and first quartile in Figure 4-that greater uncertainty ${ }_{\mathrm{PCS}}$ will translate into a carbon budget lower than the mean expectation, because uncertainty $_{B}$ in the budget increases in sync with uncertainty PCS $_{\text {. }}$

Finally, because mitigation costs vary with emissions trajectories, considerations about mitigation often seem to be inextricably intertwined with decisions about the appropriate economic discount rate (e.g., Tomassini et al., 2010). To sidestep the role of discounting, with the arguably subjective decisions it entails, we focused on the effects of uncertainty ${ }_{\mathrm{PCS}}$ on the likelihood of mitigation failurethat is, the likelihood that an unachievably rapid cessation of emissions is required in order to keep warming below an agreed limit of $2^{\circ} \mathrm{C}$. This likelihood of mitigation failure was found to increase with greater uncertainty ${ }_{\mathrm{PCS}}$. 


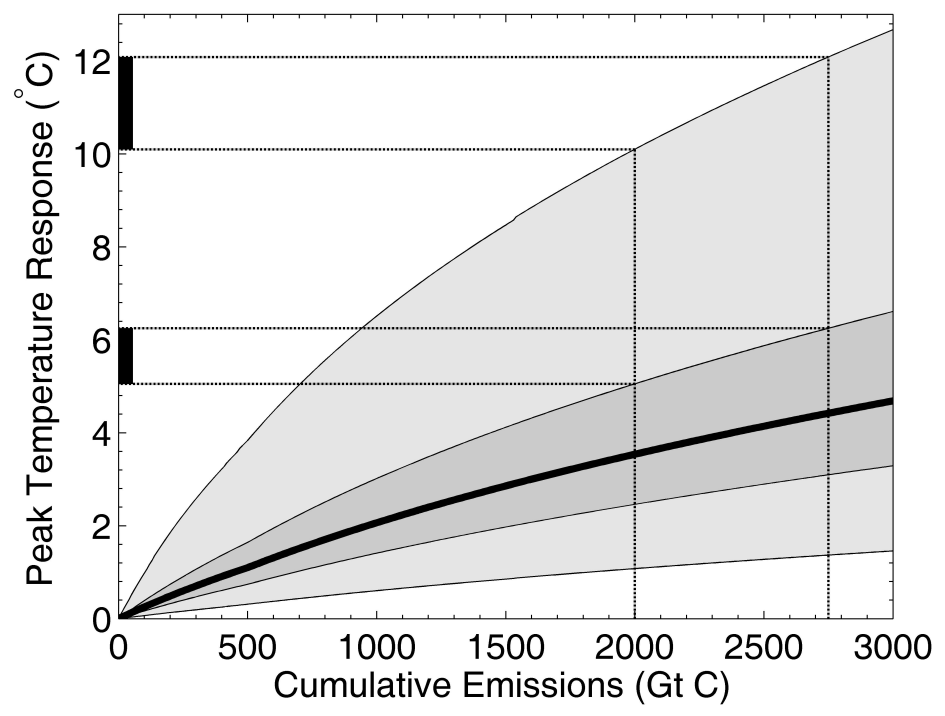

Fig. 5 Mean peak temperature response of the climate system relative to pre-industrial times as a function of cumulative carbon emissions, assuming a climate sensitivity of $\mu_{L}=2.75^{\circ} \mathrm{C}$ (solid line) and associated distribution of responses (shaded areas). Each shaded area covers the 5 th to the 95 th percentiles of the simulated distribution of temperature responses across 1,000 samples from the climate-sensitivity distribution. The darker shaded area corresponds to uncertaintyPCS $\sigma_{L}=0.52$ and the lighter area is for uncertaintyPCS $\sigma_{L}=1.66$. The dotted lines show the reduction in extreme (95th percentile) temperature responses when cumulative emissions are capped at 2,000 $\mathrm{Gt} \mathrm{C}$ as opposed to 2,750 Gt $\mathrm{C}$ : The thick vertical lines next to the ordinate permit comparison in the reduction of that extreme risk between the two levels of uncertainty ${ }_{\mathrm{PCS}}$. The figure is created using Equations 8 and 13 in Raupach et al. (2011); all forcings are considered.

\section{Reducing the Leverage of Uncertainty}

A final implication of climate uncertainty ${ }_{\mathrm{PCS}}$ discovered here involves what we call "leverage" - i.e., the ultimate impact on the climate system arising from a given climate sensitivity. Figure 5 illustrates this notion by again showing the expected temperature response of the climate system as a function of cumulative carbon emissions for a mean sensitivity $\mu_{L}=2.75^{\circ} \mathrm{C}$. As cumulative emissions increase, so does their impact on the climate system; emissions thus "leverage" the response to a constant sensitivity.

The figure also provides information about the distribution of possible climate responses when climate sensitivity is sampled from a distribution with constant mean $\left(\mu_{L}=2.75^{\circ} \mathrm{C}\right)$ and standard deviation $\sigma_{L}=0.52^{\circ} \mathrm{C}$ (dark shaded area) or $\sigma_{L}=1.66^{\circ} \mathrm{C}$ (light shaded area). Each shaded area in the figure encloses the 5 th through the 95 th percentile of the distribution of 1,000 sampled temperature responses, thus graphically characterizing uncertainty

The shaded areas invite several observations: First, as uncertaintypCS increases, so does the uncertainty shaded area $\left(\sigma_{L}=1.66^{\circ} \mathrm{C}\right)$ encloses a greater range of temperatures than the darker area $\left(\sigma_{L}=0.52^{\circ} \mathrm{C}\right)$. This is entirely unsurprising because, as noted in 
connection with Figure 2, the temperature response is merely a multiple (or fraction) of the sensitivity scaled by the total emissions. Second, the areas of uncertaintyтR are asymmetrical: Irrespective of the level of uncertaintyPCS, the area above the mean expected temperature response (solid line) is greater than the area below it. ${ }^{6}$ This asymmetry is a direct consequence of the fact that the lognormal sensitivity distribution has a fat upper tail. Third, again irrespective of the level of uncertainty ${ }_{\mathrm{PCS}}$, the vertical extent of the areas increases with cumulative emissions. That is, for a constant climate sensitivity (constant $\mu_{L}$ ) and constant uncertainty ${ }_{\mathrm{PCS}}$ in that estimate (constant $\sigma_{L}$ ), the resultant uncertainty ${ }_{\mathrm{TR}}$ in the global temperature response increases with total emissions. The latter point bears further exploration because it clarifies the notion of leverage: Even if the parameters of the climate system itself, captured by $\mu_{L}$ and $\sigma_{L}$, remain invariant, the uncertaintyтr in the overall outcome - namely, the global temperature response - increases together with cumulative emissions. Emissions thus leverage the uncertainty in the climate system.

However, by the same token, Figure 5 also offers a path towards averting the greater damages from climate change that are known to be associated with greater uncertainty (Lewandowsky et al., in press): By limiting cumulative emissions, uncertaintyтR about the climate response is reduced together with the mean expected warming. This result is simply the flipside of the fact that emissions leverage uncertainty ${ }_{\mathrm{PCS}}$.

Moreover, the reduction of uncertainty ${ }_{\mathrm{TR}}$ by limiting emissions is proportional to the absolute level of uncertaintyPCS: The greater the uncertaintypCS about climate sensitivity (light vs. dark shaded areas in Figure 5), the greater the reduction in the risk of extremely high temperature responses (i.e., at the 95th percentile) that can be achieved by limiting emissions. This effect can be seen by comparing the height of the thick vertical line adjacent to $6^{\circ} \mathrm{C}$ on the $Y$-axis with that adjacent to $10^{\circ}$. Each thick line represents the reduction in the "worst-case" (i.e., 95th percentile) temperature response when cumulative emissions are capped at 2,000 rather than $2,750 \mathrm{Gt} \mathrm{C}$ : that reduction in extreme risk is visibly greater with the higher level of uncertainty ${ }_{\mathrm{PCS}}$. Limiting emissions is therefore not only particularly advisable when uncertainty is greater, but also particularly effective in reducing extreme risks.

\section{Potential Limitations and Contrasts With Other Approaches}

Critics might argue that our approach is a thinly disguised version of the precautionary principle, thereby inheriting all its problems (e.g., vagueness, focus on adverse outcomes of one policy option only). However, unlike the precautionary principle, our approach is symmetrical as we consider the impact of uncertainty on costs (or risks) on both unabated carbon emissions (Lewandowsky et al., in press)

6 This asymmetry is exacerbated by the fact that the area below the mean estimate is even greater in our simulation than can be expected in reality: This arises from the intention to keep mean sensitivity $\left(\mu_{L}\right)$ constant across levels of uncertainty ${ }_{\mathrm{PCS}}\left(\sigma_{L}\right)$ for our illustrative purposes. In reality, the lower bound of possible climate sensitivities is quite well known and values below $1.5^{\circ} \mathrm{C}$ are considered implausible (Meehl et al., 2007). If our simulated distributions of sensitivity were truncated at that lower bound, the asymmetry of the areas representing uncertainty ${ }_{\mathrm{TR}}$ would be further enhanced. 
and on mitigation efforts. Second, unlike some interpretations of the precautionary principle, we operationalized uncertainty in a circumscribed and quantitatively informed manner.

A related criticism might hold that, just like cost-benefit analysis (CBA), our approach relies on tacit ethical judgments and that therefore nothing is gained over and above conventional CBA. In response we note that there are several assumptions that are required by CBA but not by our approach. First, our approach does not require any assumptions about monetarization of goods such as species diversity. Second, because our approach focuses on the failure rate of mitigation rather than any absolute costs, our conclusions regarding mitigation are necessarily invariant with respect to the discount rate. If mitigation fails because emissions must be cut with greater speed than what technology can possibly deliver, the discount rate is irrelevant. Likewise, our ordinal consideration of uncertainty and damage costs in the companion paper (Lewandowsky et al., in press) also side-stepped the role of the discount rate because greater damages are likely accelerated relative to lesser ones.

Our approach does, however, suffer from one drawback compared to CBA or the precautionary principle: Unlike CBA or any extant economic modeling, we cannot deliver quantitative conclusions that go beyond the ordinal level of comparison. That is, we can identify the consequences of greater uncertainty, and in doing so can point to emissions reductions as a risk-minimization pathway (see discussion surrounding Figure 5), but we cannot specify a precise quantity, such as the "social cost of carbon" (Tol, 2011, p. 419). Similarly, by focusing on a binary outcome relating to whether or not a threshold (e.g., $2^{\circ} \mathrm{C}$ ) is crossed, we collapse a range of possible outcomes into a single "failure" category that ignores the difference between a peak warming of $2.01^{\circ} \mathrm{C}$ and $4^{\circ} \mathrm{C}$ or even $6^{\circ} \mathrm{C}$. We acknowledge these limitations and offer two considerations in response: First, for the reasons noted by Lewandowsky et al. (in press), the quantitative precision of economic modeling and CBA (e.g., \$59/ton; Nordhaus, 2010) nonetheless incorporates subjective considerations. Second, even though we focused on a binary threshold-related outcome, our analysis entails a cardinal component. Specifically, if the realizations of climate sensitivity that led to mitigation failures in Figure 4 are analyzed further, the mean sensitivity of those failed realizations increases together with uncertainty ${ }_{\mathrm{PCS}}$ : Specifically, when $\sigma_{L}=0.52^{\circ} \mathrm{C}$, the mean of the sensitivities that entailed mitigation failures was $5.40^{\circ} \mathrm{C}$, and for $\sigma_{L}=1.66^{\circ} \mathrm{C}$, the mean was $6.49^{\circ} \mathrm{C}$. Put another way, greater likelihood of mitigation failure was associated with a greater expected peak temperature response.

\section{Conclusions}

We examined the consequences of uncertainty in our knowledge about future climate change on the decision whether or not to cut greenhouse emissions. Our analysis focused on the ordinal relationship between uncertainty PCS $_{\text {and }}$ the probability of adverse outcomes associated with the two principal policy options-viz. to continue emitting greenhouse gases or to mitigate climate change by cutting emissions. Exploration of both options yielded the result that greater uncertaintypCS necessarily translates into greater risk. 
First, as uncertainty about climate sensitivity increases, so does the damage expected from unmitigated climate change. The details of this analysis can be found in Lewandowsky et al. (in press); to summarize briefly, this result arises as a necessary consequence when the function that relates warming to expected damages is convex, as is assumed by extant economic models. The result also arises without any assumptions about the economic damage function when uncertainty about future sea level rise is considered: It is a consequence of simple extremevalue statistics that increasing uncertainty about sea level rise translates into an increasingly greater protective allowance that is required to keep the risk from flooding constant.

Second, we have shown in this article that as uncertainty ${ }_{\mathrm{PCS}}$ increases, so does the likelihood that mitigation efforts will fail. In order to have a chance to limit warming to a predetermined upper bound (e.g., $2^{\circ} \mathrm{C}$ ), total cumulative emissions, from the beginning of industrialization onward, must not exceed a set budget. If the total size of that budget is less than or equal to the historical emissions already incurred, then mitigation has already failed. By that criterion, the probability of mitigation failure is an increasing function of uncertainty in our knowledge of climate sensitivity.

Thus, greater uncertainty ${ }_{\mathrm{PCS}}$ implies greater damage from unmitigated climate change and it also implies a greater likelihood of the failure of mitigation attempts. The converse also holds: If uncertainty ${ }_{\mathrm{PCS}}$ could be reduced, this would alleviate the risk of damages as well as the risk of mitigation failure. Our analysis revealed a path to reducing the risks generated by uncertainty ${ }_{\mathrm{PCS}}$ based on the fact that those risks are "leveraged" by cumulative greenhouse gas emissions. As cumulative emissions increase, so does uncertainty $\mathrm{TR}_{\mathrm{R}}$ in the overall temperature response, even if the parameters of the climate system remain unchanged. By the same token, a reduction in emissions reduces the leverage of uncertainty ${ }_{\mathrm{PCS}}$, thereby reducing the adverse impacts of climate uncertainty.

Contrary to the claim by some researchers that uncertainty presents a barrier to scientifically-informed policy decisions (Allenby \& Sarewitz, 2011; Sarewitz, 2004), any appeal to scientific uncertainty actually implies a stronger, rather than weaker, need to cut greenhouse gas emissions than in the absence of uncertainty.

\section{References}

Allenby, B. R., \& Sarewitz, D. (2011). We've made a world we cannot control. New Scientist, 210(2812), 28-29.

Annan, J. D., \& Hargreaves, J. C. (2006). Using multiple observationally-based constraints to estimate climate sensitivity. Geophysical Research Letters, 33, L06704.

Brownell, K. D., \& Warner, K. E. (2009). The perils of ignoring history: Big tobacco played dirty and millions died. How similar is big food? Milbank Quarterly, 87, 259-294. doi: 10.1111/j.1468-0009.2009.00555.x

Freudenburg, W. R., Gramling, R., \& Davidson, D. J. (2008). Scientific certainty argumentation methods (SCAMs): Science and the politics of doubt. Sociological Inquiry, 78, 2-38.

Freudenburg, W. R., \& Muselli, V. (2013). Reexamining climate change debates: Scientific disagreement or scientific certainty argumentation meth- 
ods (SCAMs)? American Behavioral Scientist, 57, 777-795. doi: 10.1177/ 0002764212458274

Garnaut, R. (2011). The Garnaut review 2011: Australia in the global response to climate change. Cambridge: Cambridge University Press.

Hunter, J. (2012). A simple technique for estimating an allowance for uncertain sea-level rise. Climatic Change, 113, 239-252. doi: 10.1007/s10584-011-0332 $-1$

Intergovernmental Panel on Climate Change. (2005). Guidance notes for lead authors of the IPCC Fourth Assessment Report on addressing uncertainties. (Tech. Rep.).

Jahn, D., \& Korolczuk, S. (2012). German exceptionalism: the end of nuclear energy in Germany! Environmental Politics, 21, 159-164.

Jensen, J. L. W. V. (1906). Sur les fonctions convexes et les inégalités entre les valeurs moyennes. Acta Mathematica, 30, 175-193.

Kahan, D. M., Braman, D., Slovic, P., Gastil, J., \& Cohen, G. (2009). Cultural cognition of the risks and benefits of nanotechnology. Nature Nanotechnology, 4, 87-90.

Kahan, D. M., Slovic, P., Braman, D., \& Gastil, J. (2006). Laws of fear: Beyond the precautionary principle. Harvard Law Review, 119, 1071-1109.

Kim, K. S. (2011). Public understanding of the politics of global warming in the news media: the hostile media approach. Public Understanding of Science, 20, 690-705. doi: 10.1177/0963662510372313

Lewandowsky, S., Risbey, J. S., Smithson, M., Newell, B. R., \& Hunter, J. (in press). Scientific uncertainty and climate change: Part I. Uncertainty and unabated emissions. Climatic Change.

Meehl, G. A., Stocker, T. F., Collins, W. D., Friedlingstein, P., Gaye, A. T., Gregory, J. M., ... Zhao, Z.-C. (2007). Global climate projections. In S. Solomon et al. (Eds.), Climate change 2007: The physical science basis. contribution of working group I to the Fourth Assessment Report of the Intergovernmental Panel on Climate Change (pp. 748-845). Cambridge: Cambridge University Press.

Michaels, D., \& Monforton, C. (2005). Manufacturing uncertainty: Contested science and the protection of the public's health and environment. American Journal of Public Health, 95, S39-S48. doi: 10.2105/AJPH.2004.043059

Narita, D. (2012). Managing uncertainties: The making of the IPCCs Special Report on Carbon Dioxide Capture and Storage. Public Understanding of Science, 21, 84-100.

Nilsson, M., Beaglehole, R., \& Sauerborn, R. (2009). Climate policy: lessons from tobacco control. Lancet, 374, 1955-1956.

Nisbet, M. C. (2009). Framing science: A new paradigm in public engagement. In L. Kahlor \& P. Stout (Eds.), Understanding science: New agendas in science communication (pp. 40-67). New York: Taylor \& Francisb.

Nordhaus, W. D. (2010). Economic aspects of global warming in a postCopenhagen environment. Proceedings of the National Academy of Science, 107, 11721-11726.

Oreskes, N., \& Conway, E. M. (2010). Merchants of doubt. London: Bloomsbury Publishing.

Pacala, S., \& Socolow, R. (2004). Stabilization wedges: Solving the climate problem for the next 50 years with current technologies. Science, 305, 968-972. 
Padilla, L. E., Vallis, G. K., \& Rowley, C. W. (2011). Probabilistic estimates of transient climate sensitivity subject to uncertainty in forcing and natural variability. Journal of Climate, 24, 5521-5537. doi: 10.1175/2011JCL13989.1

Proctor, R. N. (2004). Should medical historians be working for the tobacco industry? Lancet, 363, 1174-1175.

Proctor, R. N. (2008). On playing the Nazi card. Tobacco Control, 17, 289-290.

Raupach, M. R. (2013). The exponential eigenmodes of the carbon-climate system, and their implications for ratios of responses to forcings. Earth System Dynamics, 4, 31-49.

Raupach, M. R., Canadell, J. G., Ciais, P., Friedlingstein, P., Rayner, P. J., \& Trudinger, C. M. (2011). The relationship between peak warming and cumulative CO2 emissions, and its use to quantify vulnerabilities in the carbonclimate-human system. Tellus, 63B, 145-164.

Roe, G. H., \& Baker, M. B. (2007). Why is climate sensitivity so unpredictable? Science, 318, 629-632.

Rogelj, J., Hare, W., Lowe, J., van Vuuren, D. P., Riahi, K., Matthews, B., ... Meinshausen, M. (2011). Emission pathways consistent with a $2{ }^{\circ} \mathrm{C}$ global temperature limit. Nature Climate Change. doi: 10.1038/NCLIMATE1258

Sarewitz, D. (2004). How science makes environmental controversies worse. Environment Science 83 Policy, 7, 385-403.

Slovic, P. (1987). Perception of risk. Science, 236, 280-285.

Slovic, P. (1999). Trust, emotion, sex, politics, and science: Surveying the riskassessment battlefield. Risk Analysis, 19, 689-701.

Smith, P., Bansal-Travers, M., O’Connor, R., Brown, A., Banthin, C., GuardinoColket, S., \& Cummings, K. (2011). Correcting over 50 years of tobacco industry misinformation. American Journal of Preventive Medicine, 40, 690698.

Smith, S. M., Lowe, J. A., Bowerman, N. H. A., Gohar, L. K., Huntingford, C., \& Allen, M. R. (2012). Equivalence of greenhouse-gas emissions for peak temperature limits. Nature Climate Change, 2, 535-538. doi: 10.1038/NCLIMATE1496

Socolow, R. (2011, 27 September). Wedges reaffirmed. Bulletin of the Atomic Scientists, (web edition).

Solomon, S., Plattner, G.-K., Knutti, R., \& Friedlingstein, P. (2009). Irreversible climate change due to carbon dioxide emissions. Proceedings of the National Academy of Sciences of the United States of America, 106, 1704-1709. doi: $10.1073 /$ pnas.0812721106

Stern, N. (2007). The economics of climate change: The Stern review. Cambridge: Cambridge University Press.

Sterner, T., \& Persson, U. M. (2008). An even Sterner review: Introducing relative prices into the discounting debate. Review of Environmental Economics and Policy, 2, 61-76.

Tol, R. S. J. (2011). The social cost of carbon. Annual Review of Resource Economics, 3, 419-443.

Tomassini, L., Knutti, R., Plattner, G.-K., van Vuuren, D. P., Stocker, T. F., Howarth, R. B., \& Borsuk, M. E. (2010). Uncertainty and risk in climate projections for the 21st century: comparing mitigation to non-intervention scenarios. Climatic Change, 103, 399-422. doi: 10.1007/s10584.009.9763.3 
Vlek, C. (2010a). Judicious management of uncertain risks: I. Developments and criticisms of risk analysis and precautionary reasoning. Journal of Risk Research, 13, 517-543.

Vlek, C. (2010b). Judicious management of uncertain risks: II. Simple rules and more intricate models for precautionary decision-making. Journal of Risk Research, 13, 545-569. doi: 10.1080/13669871003629903

Webster, M., Forest, C., Reilly, J., Babiker, M., Kicklighter, D., Mayer, M., ... Wang, C. (2003). Uncertainty analysis of climate change and policy response. Climatic Change, 61, 295-320. doi: 10.1023/B:CLIM.0000004564.09961.9f

Acknowledgements Preparation of this paper was facilitated by a Discovery Grant from the Australian Research Council, an Australian Professorial Fellowship, a Discovery Outstanding Researcher Award, and a Wolfson Research Merit Award from the Royal Society, to the first author, by a Future Fellowship from the Australian Research Council to Ben Newell, and funding from the Australian Research Council Centre of Excellence in Climate Systems Science. The work was also supported by a Linkage Grant from the Australian Research Council and a grant from the National Climate Change Adaptation Research Facility and the CSIRO Climate Adaptation Flagship. We thank four reviewers for their incisive critique and helpful comments. Correspondence to the first author at the School of Experimental Psychology, University of Bristol, 12A Priory Road, Bristol BS8 1TU, United Kingdom (stephan.lewandowsky@bristol.ac.uk). Personal web page: http://www.cogsciwa.com. 